\title{
Dynamics of atom tunnelling in a symmetric double well coupled to an asymmetric double well: The case of malonaldehyde ${ }^{\#}$
}

\author{
S GHOSH and S P BHATTACHARYYA* \\ Department of Physical Chemistry, Indian Association for the Cultivation of Science, \\ Jadavpur, Calcutta 700 032, India \\ e-mail: pcspb@iacs.res.in
}

\begin{abstract}
The quantum dynamics of intramolecular H-atom transfer in malonaldehyde is investigated with a model two-dimensional Hamiltonian constructed with the help of available ab initio theoretical data on the relevant portion of the potential energy surface. At zero temperature, the H-atom transfer takes place by tunnelling leading to cis-cis isomerization while the cis-trans channel remains closed. Local excitation of the cis-trans mode by an external field is predicted to quench cis-cis tunnelling isomerization while excitation of the cis-cis mode is found to enhance the isomerization by tunnelling.
\end{abstract}

Keywords. Intramolecular H-transfer; quenching of tunnelling; quantum phase space diagram; control of tunnelling; mean field dynamics.

\section{Introduction}

Quantum mechanical tunnelling has been a subbject of intense study ever since quantum mechanics was formulated. In fact, the phenomenon of atom tunnelling pervades different branches of physics, chemistry and biology. ${ }^{1-3}$ There are mainly three kinds of phenomena and processes that have been studied in the context of atom tunnelling in the gas phase. The energy splitting in symmetric double well potentials has been widely known to provide spectral signature of tunnelling. ${ }^{4}$ Dissociation of a metastable molecule by tunnelling through potential barriers and quantum tunnelling diffusion of a positive muon and munonium provide excellent examples of tunnelling dynamics mediated phenomena. ${ }^{5}$ tunnelling contribution to bimolecular reaction rate has also attracted a great deal of interest in the recent years. ${ }^{6}$ Proton or hydrogen atom tunnelling in symmetric or asymmetric double well potentials are ubiquitous. ${ }^{7-10}$ The zero temperature motion of the proton or hydrogen atom in the double well is often described by a tunnelling mode which may be coupled to, let us say, a bond stretching mode. The stretching mode may then provide a handle for controlling the tunnelling. ${ }^{11}$ If the stretching mode is replaced by another tunnelling mode, it is imperative to understand how the dynamics of tunnelling in one double well gets affected by coupling with the other double well and how one can exercise control over the tunnelling motion in one particular well.

In the present communication, we have undertaken an analysis of the questions posed above by numerically following the quantum dynamics of tunnelling of the aldehydic $\mathrm{H}$-atom in the malonaldehyde molecule. Experimental tunnelling splitting data together with $a b$ initio theoretical information about the relevant part of the potential energy surface on which the $\mathrm{H}$ atom transfer in malonaldehyde takes place are used to construct and calibrate a model two-dimensional potential for describing the tunnelling dynamics of malonaldehyde. A mean field method is used to describe the dynamics within the framework of time dependent Fourier Grid Hamiltonian (TDFGH) formulation ${ }^{12-14}$ and the dynamics of tunnelling is analysed in detail. We have also investigated how photo-excitation of one of the two coupled tunnelling modes affects the tunnelling dynamics of the other and can be used to control the tunnelling isomerization in malonaldehyde.

\section{Methods}

Let $H_{0}(x, y)$ be the Hamiltonian of a particle of mass ' $m$ ' moving on the $x-y$ plane in a potential $V(x, y) . V(x, y)$ represents a superposition of a symmetric double well

\footnotetext{
\#Dedicated to Prof. N Sathyamurthy on his 60th birthday

*For correspondence
} 
potential $V_{1}(x)$ and an asymmetric double well $V_{2}(y)$. Thus, we have

$$
\begin{aligned}
H_{0}(x, y) & =H_{1}(x)+H_{2}(y) \\
& =T(x)+V_{1}(x)+T(y)+V_{2}(y) .
\end{aligned}
$$

In equation (1) two tunnelling modes are represented by two independent coordinates $x$ and $y$, respectively. We assume that $H_{1}(x)$ represents the Hamiltonian describing the motion in the symmetric double well while $H_{2}(y)$ represents motion in an asymmetric double well potential. Let $V_{\text {int }}(x, y)$ represent the coupling between the two modes and $\lambda$ be the strength of the coupling. Then the total Hamiltonian is given by

$$
H_{0}(x, y)=H_{1}(x)+H_{2}(y)+\lambda V_{\text {int }}(x, y),
$$

where $H_{1}(x)$ is given by

$$
H_{1}(x)=T(x)+V_{1}(x),
$$

and

$$
H_{2}(y)=T(y)+V_{2}(y) .
$$

$V_{1}(x)$ and $V_{2}(y)$ being the symmetric and asymmetric double well potentials. Dynamics of the coupled system can be studied in a time-dependent mean field framework where we write the wave function of the composite system as

$$
|\psi(x, y, t)\rangle=\left|\phi^{x} \chi^{y}\right\rangle .
$$

In the TDFGH method, we choose

$$
\left|\phi^{x}\right\rangle=\sum_{p=1}^{n_{x}} w_{p}^{x}(t)\left|x_{p}\right\rangle \Delta x,
$$

and

$$
\left|\chi^{y}\right\rangle=\sum_{q=1}^{n_{y}} w_{q}^{y}(t)\left|y_{q}\right\rangle \Delta y,
$$

$w_{p}^{x}(t)$ and $w_{q}^{y}(t)$ are the time-dependent grid point amplitudes along the $x$ and $y$ directions, while $\left|\phi^{x}\right\rangle$ and $\left|\chi^{y}\right\rangle$ are approximate eigenfunctions of $H_{1}(x)$ and $H_{2}(y)$, respectively. Time dependent variational principle ${ }^{15,16}$ leads to the following evolution equations for $\left|\phi^{x}\right\rangle$ and $\left|\chi^{y}\right\rangle$ in the presence of the interaction potential $V_{\text {int }}(x, y)^{14}$

$$
\begin{aligned}
i \hbar \frac{\left|\phi^{x}(t)\right\rangle}{d t} & =\left(H_{1}(x)+\overline{\varepsilon_{y}}+\lambda V_{\mathrm{int}}(x, \bar{y}, t)\right)\left|\phi^{x}\right\rangle \\
& =H_{\mathrm{eff}}(x, t)\left|\phi^{x}(t)\right\rangle
\end{aligned}
$$

and

$$
\begin{aligned}
\mid i \hbar \frac{\left|\chi^{y}(t)\right\rangle}{d t} & =\left(H_{2}(y)+\overline{\varepsilon_{x}}+\lambda V_{\mathrm{int}}(\bar{x}, y, t)\right)\left|\chi^{y}\right\rangle \\
& =H_{\mathrm{eff}}(y, t)\left|\chi^{y}(t)\right\rangle,
\end{aligned}
$$

$V_{\text {int }}(x, \bar{y}, t)$ is the effective time-dependent potential spatially averaged over $y$ at time $t$ and acting along the $x$ direction while $V_{\text {int }}(\bar{x}, y, t)$ is the $x$-averaged counterpart of the interaction potential for the motion along the $y$ direction. With reference to the above mentioned equations time evolution of the grid point amplitudes are easily obtained by projecting equations (8) and (9) with $\left\langle x_{p}(t)\right|$ and $\left\langle y_{q}(t)\right|$, respectively and are given by

$$
\begin{aligned}
i \hbar \dot{w}_{p}^{x}(t) & =\sum_{q=1}^{n_{x}}\left\langle x_{p}\left|H_{\mathrm{eff}}(x, t)\right| x_{q}\right\rangle w_{q}^{x}(t), \\
p & =1,2, \ldots ., n_{x}
\end{aligned}
$$

and

$$
\begin{aligned}
i \hbar \dot{w}_{q}^{y}(t) & =\sum_{r=1}^{n_{x}}\left\langle y_{q}\left|H_{\mathrm{eff}}(y, t)\right| y_{r}\right\rangle w_{r}^{y}(t), \\
q & =1,2, \ldots . ., n_{y} .
\end{aligned}
$$

These equations can be numerically integrated by a suitable integrator like the sixth order Runge-Kutta method.

When a quantum particle moves in a symmetric double well potential there is a non-zero probability of its tunnelling from one well to the other even if its energy is less than the energy required to reach the barrier top. Let us suppose that the particle was initially localized in the left well of the uncoupled symmetric doublewell (figure 1a) potential (cis-cis mode). The lowest energy localized states $\left(\phi_{L}, \phi_{R}\right)$ with this potential, can be described by linear combinations of the two lowest energy eigen-states of even $\left(\psi_{0}^{+}\right)$and odd parity $\left(\psi_{0}^{-}\right)$, respectively:

$$
\begin{aligned}
& \phi_{L}=\frac{1}{\sqrt{2}}\left(\psi_{0}^{+}+\psi_{0}^{-}\right), \\
& \phi_{R}=\frac{1}{\sqrt{2}}\left(\psi_{0}^{+}-\psi_{0}^{-}\right),
\end{aligned}
$$

where $\phi_{L}$ and $\phi_{R}$ represent the states localized in the left and the right well, respectively. In the absence of any coupling between cis-cis (symmetric double well) and the cis-trans (asymmetric double well) isomerization modes the particle tunnels coherently from the left well to the right well. If one takes $\phi_{L}$ to be the initial state, tunnelling probability is obtained by calculating the probability of finding the particle in the right well. 


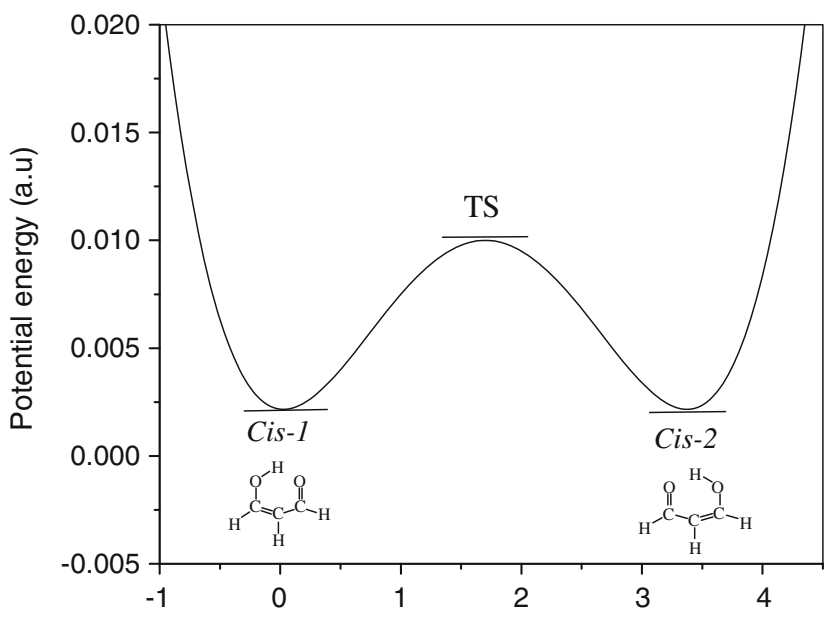

(a)

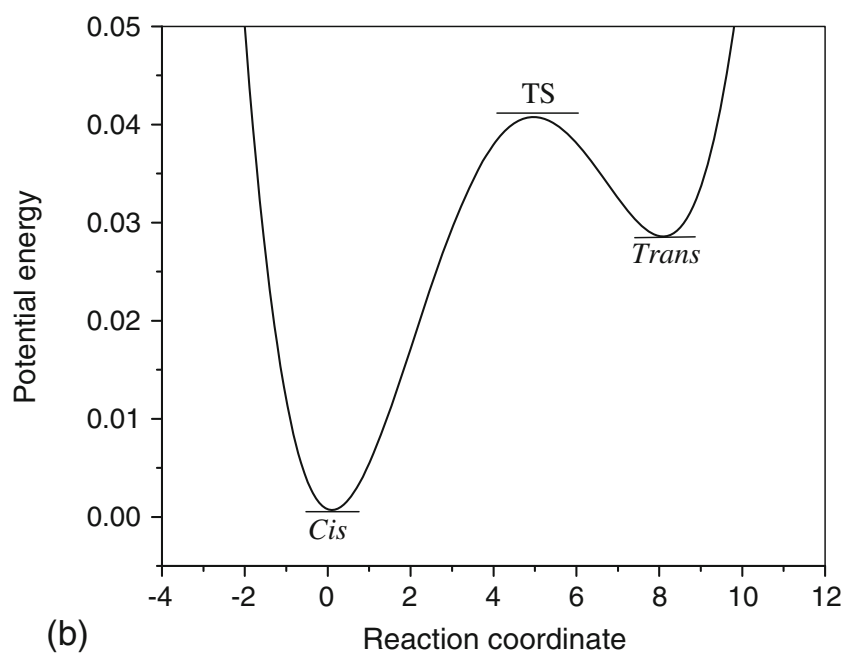

Figure 1. (a) Potential energy surface along $c i s \leftrightarrow c i s$ reaction coordinate. (b) Potential energy surface along cis $\leftrightarrow$ trans reaction coordinate.

The probability $P_{R}(t)$ at any particular instant is given by (the barrier is located at $x=0$ )

$$
P_{R}(t)=\int_{0}^{\infty}|\psi(x, t)|^{2} d x,
$$

which, in the two-dimensional case (using mean field formalism ${ }^{14}$ and the Fourier Grid Hamiltonian (FGH) ${ }^{12}$ basis) translates into

$$
P_{R}(t)=\sum_{p=\frac{n_{x}-1}{2}}^{n_{x}} \sum_{q=1}^{n_{y}} w_{p}^{x *} w_{q}^{y} \Delta x \Delta y .
$$

The rate of tunnelling may be obtained by computing the average slope of the $P_{R}(t)-t$ graph. The tunnelling rate can also be calculated from the average slope of the $\langle x(t)\rangle-t$ graph which leads to average tunnelling velocity $\langle v\rangle$. The tunnelling time is obtained by setting $\tau_{\text {tunnelling }}=\frac{l_{0}}{\langle v\rangle}$, where $l_{0}$ is the idealized well-to-well distance. Inverse of $\tau_{\text {tunnelling }}$ leads to the tunnelling rate. ${ }^{17}$ To compute $\langle x(t)\rangle$, we evaluate the expression

$$
\langle x(t)\rangle=\langle\psi(x, y, t)|x| \psi(x, y, t)\rangle .
$$

Substituting the expansion for $|\psi(x, y, t)\rangle$ from equation (4) in equation (16) we get

$$
\langle x(t)\rangle=\sum_{p}^{n_{x}} w_{p}^{x *} x_{p} w_{p}^{x} .
$$

The rate of tunnelling is also related to the tunnelling splitting in the symmetric double well. When the symmetric double well gets coupled to an asymmetric double well the tunnelling splitting or rate can increase or decrease or stay unaffected depending upon the nature and strength of the coupling which in turn is determined by the functional form of $V_{\text {int }}(x, y)$ and the coupling strength $\lambda .{ }^{11}$ We can also calculate the tunnelling rate or probability when one of the coupled modes is excited by a time varying external field by following the procedure already outlined. Thus a time-dependent sinusoidally varying electric field applied along the $y$ direction, leads to the following sets of evolution equations for $w_{p}^{x}(t), w_{q}^{y}(t)$

$$
\begin{aligned}
i \hbar \dot{w}_{p}^{x}(t) & =\sum_{q=1}^{n_{x}}\left\langle x_{p}\left|H_{\text {eff }}(x, t)\right| x_{q}\right\rangle w_{q}^{x}(t), \\
p & =1,2, \ldots . . n_{x}
\end{aligned}
$$

and

$$
\begin{aligned}
i \hbar \dot{w}_{q}^{y}(t) & =\sum_{r=1}^{n_{x}}\left\langle y_{q}\left|H_{\mathrm{eff}}^{\prime}(y, t)\right| y_{r}\right\rangle w_{r}^{y}(t), \\
q & =1,2, \ldots . . n_{y}
\end{aligned}
$$

where

$$
H_{\text {eff }}^{\prime}(y, t)=H_{\text {eff }}(y, t)+\varepsilon_{y}^{0} y \sin (\omega t+\delta)
$$

$H_{\text {eff }}(x, t)$ and $H_{\text {eff }}(y, t)$ have already been defined in equation (8) and equation (9), respectively.

\section{Results and discussion}

\subsection{Constructing the model potential}

It has been suggested that the ground state intramolecular $\mathrm{H}$-atom transfer in malonaldehyde molecule takes place via tunnelling. ${ }^{18}$ The global cis-to-cis $\mathrm{H}$-atom transfer occurs via a low energy planar transition state. The section of potential energy surface (along $x$ ) on which the transfer takes place can be described by a symmetric double well potential $V_{1}(x)$ (figure 1a), $x$ 
representing the in-plane motion of the tunnelling $\mathrm{H}$ atom. The cis to trans enolization, on the other hand, is mediated by a nonplanar high energy transition state (figure 1b). The corresponding section of the potential energy surface (along $y$ ) is described by an asymmetric double well $V_{2}(y), y$ representing the out-of-plane motion of the H-atom. We represent the effective twodimensional PES on which the $\mathrm{H}$-atom transfer takes place by $V(x, y)$ where $V(x, y)=V_{1}(x)+V_{2}(y)+$ $\lambda V_{\text {int }}(x, y)$ and assume that $V_{\text {int }}(x, y)$ is representable as $x^{m} y^{n}$. So the total Hamiltonian of the coupled system is $H(x, y)=T(x)+V_{1}(x)+T(y)+V_{2}(y)+\lambda x^{m} y^{n}$ and the effective two-dimensional PES in which the $\mathrm{H}$ atom transfer takes place is $V(x, y)=V_{1}(x)+V_{2}(y)+$ $\lambda x^{m} y^{n}$. We assume that the symmetric and asymmetric double well potentials are $V_{1}(x)=A x^{4}-B x^{2}$ (figure 1a) and $V_{2}(y)=C(y-b)^{4}-D(y-b)^{3}-E(y-$ $b)^{2}+F(y-b)$ (figure $1 \mathrm{~b}$ ), respectively. The parameters $\mathrm{A}, \mathrm{B}, \mathrm{C}, \mathrm{D}, \mathrm{E}$ and $\mathrm{F}$ have been determined so that the locations and the depths of the global cis, and local trans minima and the barrier heights match as closely as possible with the accurate theoretical predictions of Yagi et al ${ }^{18}$ made on the basis of MP2 6-31G (d,p) calculation. The magnitude of the shift ' $b$ ' used in $V_{2}(y)$ is 2.4 a.u. The parameters A and B were fixed from the known barrier height of the cis1-cis2 transfer and location of the TS of the process as predicted by Yagi et al. ${ }^{18}$ The parameters $\mathrm{C}, \mathrm{D}, \mathrm{E}$ and $\mathrm{F}$ were fixed by using the available information on the location and the energy of the trans conformation and the energy for the cis to trans isomerization. ${ }^{18}$ The relevant quantities used in fitting are reported in table 1 while the fitted potential parameters (A-G) are reported in table 2 .

For fixing the coupling strength $\lambda$, we have proceeded in the following manner. The Hamiltonian $H_{1}(x)$ was diagonalized in the coordinate basis $\left(\mid x_{q}>\right)$ on a grid of length of 10 a.u. containing 151 grid points while $\mathrm{H}_{2}(y)$ was similarly diagonalized on a grid of 20 a.u. containing 151 grid points by using the FGH recipe. ${ }^{12}$ Eighteen of the lowest eigenstates $\left\{\phi_{i}(x)\right\}$ of $H_{1}(x)$ and $\left\{\phi_{j}(y)\right\}$ of $H_{2}(y)$ were taken to form the

Table 1. Ab initio theoretical data used in fixing the model potential parameters.

\begin{tabular}{lcc}
\hline & $\begin{array}{c}\text { Location on the reaction } \\
\text { coordinate }(\mathrm{a}, \mathrm{u})\end{array}$ & $\begin{array}{c}\text { Energy } \\
(\mathrm{Kcal} / \mathrm{mol})\end{array}$ \\
\hline cis- 1 & 0 & 0.0 \\
cis- 2 & 3.2 & 0.0 \\
TS for cis- $1 \leftrightarrow$ cis- 2 & 1.6 & 3.6 \\
TS for cis- $1 \leftrightarrow$ trans & 4 & 19.0 \\
trans & 8 & 14.1 \\
\hline
\end{tabular}

Table 2. Double well parameters used in the calculations.

\begin{tabular}{lc}
\hline Parameters & Values (a.u.) \\
\hline A & 0.001 \\
B & 0.0056 \\
C & 0.00009374 \\
D & 0.00109 \\
E & 0.00299 \\
F & 0.005232 \\
\hline
\end{tabular}

product basis states $\left\{\phi_{i}(x) \chi_{j}(y)\right\}_{i j}$. The total Hamiltonian $H(x, y, \lambda)=H_{1}(x)+H_{2}(y)+\lambda x^{m} y^{n}$ was then diagonalized in the product basis for different values of $\lambda, m$ and $n$. The tunnelling splitting $\Delta_{0}(\lambda)$ in each case was calculated from the appropriate eigenvalues so obtained. The coupling strength $\lambda$ was chosen so as
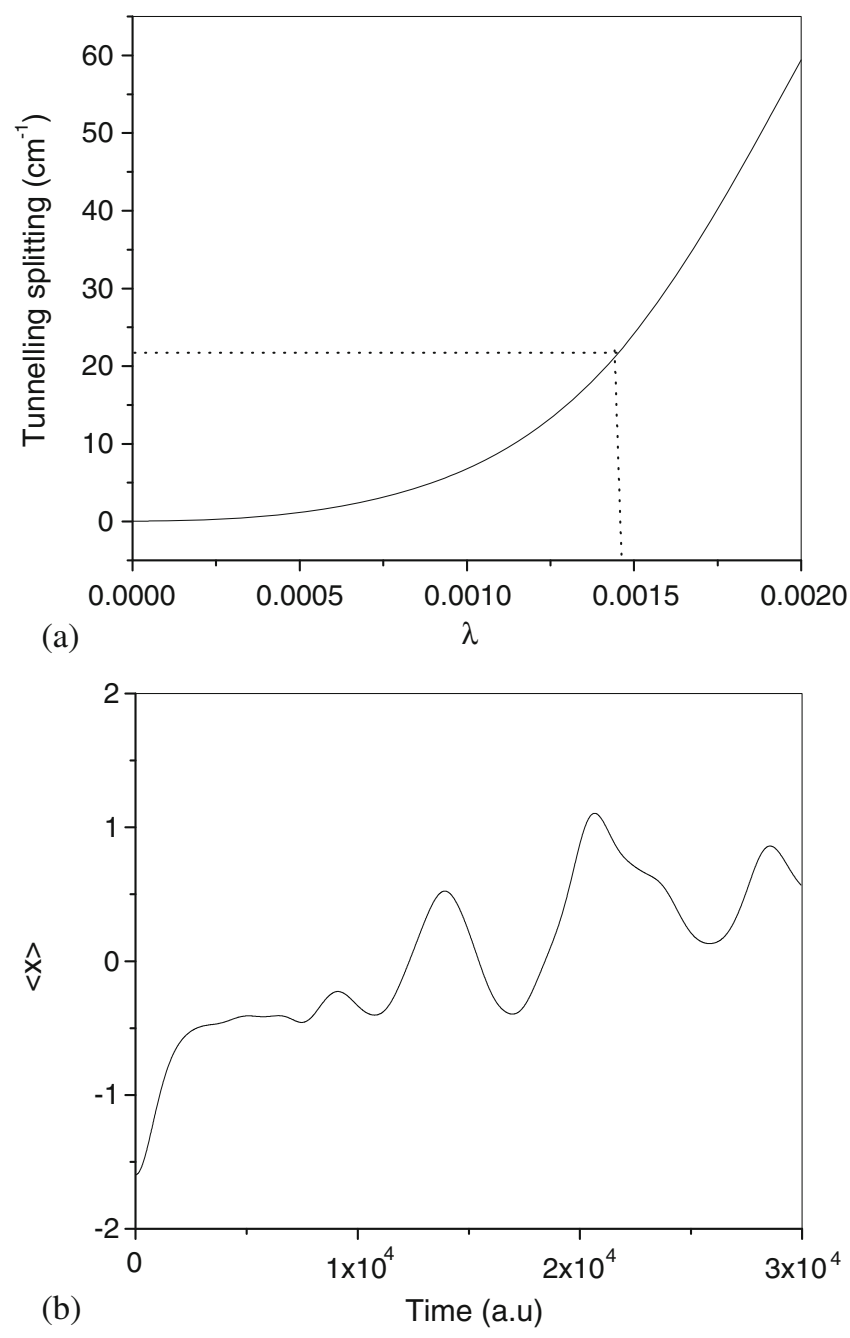

Figure 2. (a) Predicted tunnelling splitting as a function of coupling strength $\lambda$. (b) Profile of $\langle x\rangle$ versus time along the cis-cis reaction coordinate. 
Table 3. Predicted tunnelling splitting in malonaldehyde and deuterium substituted malonaldehyde compared with available experimental data $\left(\mathrm{cm}^{-1}\right)$.

\begin{tabular}{lcc}
\hline $\begin{array}{l}\text { Parameters for model } \\
\text { potential }\end{array}$ & $\begin{array}{c}\text { Predicted tunnelling } \\
\text { splitting }\end{array}$ & $\begin{array}{c}\text { Experimental } \\
\text { splitting }\end{array}$ \\
\hline$m=2, n=2, \lambda=0.0015$ & $21.5(\mathrm{H}), 2.3(\mathrm{D})$ & - \\
$m=1, n=1, \lambda=1.75^{*} 10^{-5}$ & $21.4(\mathrm{H}), 18.6(\mathrm{D})$ & $21.6(\mathrm{H})$ \\
$m=2, n=1, \lambda=1.0^{*} 10^{-5}$ & $21.4(\mathrm{H}), 19.7(\mathrm{D})$ & $2.9(\mathrm{D})$ \\
\hline
\end{tabular}

reproduce the experimental value of tunnelling splitting $\Delta_{0}$. Figure 2 a shows how the computed $\Delta_{0}$ varies with $\lambda$ when we chose $m=n=2$. The value of $\Delta_{0}(\lambda)$ for which the experimental value of $\Delta_{0}$ is reproduced is reported in (table 3). For $m=n=1$ or, $m=1$, $n=2$ the corresponding values of $\lambda_{0}$ for which the predicted value of $\Delta_{0}$, matches with experimental tunnelling splitting data are also reported in the same table. For $m=1, n=2$ we used a symmetric form of coupling i.e., $V_{\text {int }}(x, y)=\lambda\left(x^{m} y^{n}+x^{n} y^{m}\right)$ to complete the determination of the best model PES for studying tunnelling dynamics of malonaldehyde.

We have also calculated $\Delta_{0}$ for the deuterium substituted malonaldehyde by using the already calibrated potential for malonaldehyde. Table 3 reports and compares theoretically obtained values of $\Delta_{0}$ and the experimentally determined values $\Delta_{0}$ for malonaldehyde and deuterium-substituted malonaldehyde with their theoretical counterparts for three model potentials proposed by us. The agreement with experimental splitting data for malonaldehyde as well as deuterium-substituted malonaldehyde is excellent only when $V_{\text {int }}(x, y)=$ $\lambda x^{m} y^{n}$ with $m=n=2$ is used. We have therefore chosen the model Hamiltonian for describing the tunnelling dynamics of $\mathrm{H}$-atom transfer of malonaldehyde accordingly.

\subsection{2-d tunnelling dynamics in malonaldehyde}

We have performed time-dependent two-dimensional mean field calculations ${ }^{13}$ for the tunnelling $\mathrm{H}$-atom transfer in the ground state of malonaldehyde. The initial state was chosen to be $\left|\phi_{0}(x) \chi_{0}(y)\right\rangle$, where, $\left|\phi_{0}(x)\right\rangle$ and $\left|\chi_{0}(y)\right\rangle$ are the lowest eigen-states of $H_{1}(x)$ and $H_{2}(y)$, respectively. Figure $2 \mathrm{~b}$ shows how the computed $<x(t)>$ changes with time. It can be clearly seen that in the ground state (zero temperature), tunnelling results in $c i s \leftrightarrow c i s$ isomerization. The 'quantum phase space' along the corresponding coordinate is open and the 'trajectory' (figure 3a) represents underbarrier crossing from one cis structure to its degenarate counterpart, the energy remaining under the barrier through out the dynamics. The 'quantum phase space' along the cis-trans $\mathrm{H}$-atom transfer coordinate shows that the 'quantum phase space' is closed and confined to the cis side of the barrier (figure $3 b$ ). At zero temperature, therefore only the $c i s \leftrightarrow c i s$ isomerization by tunnelling
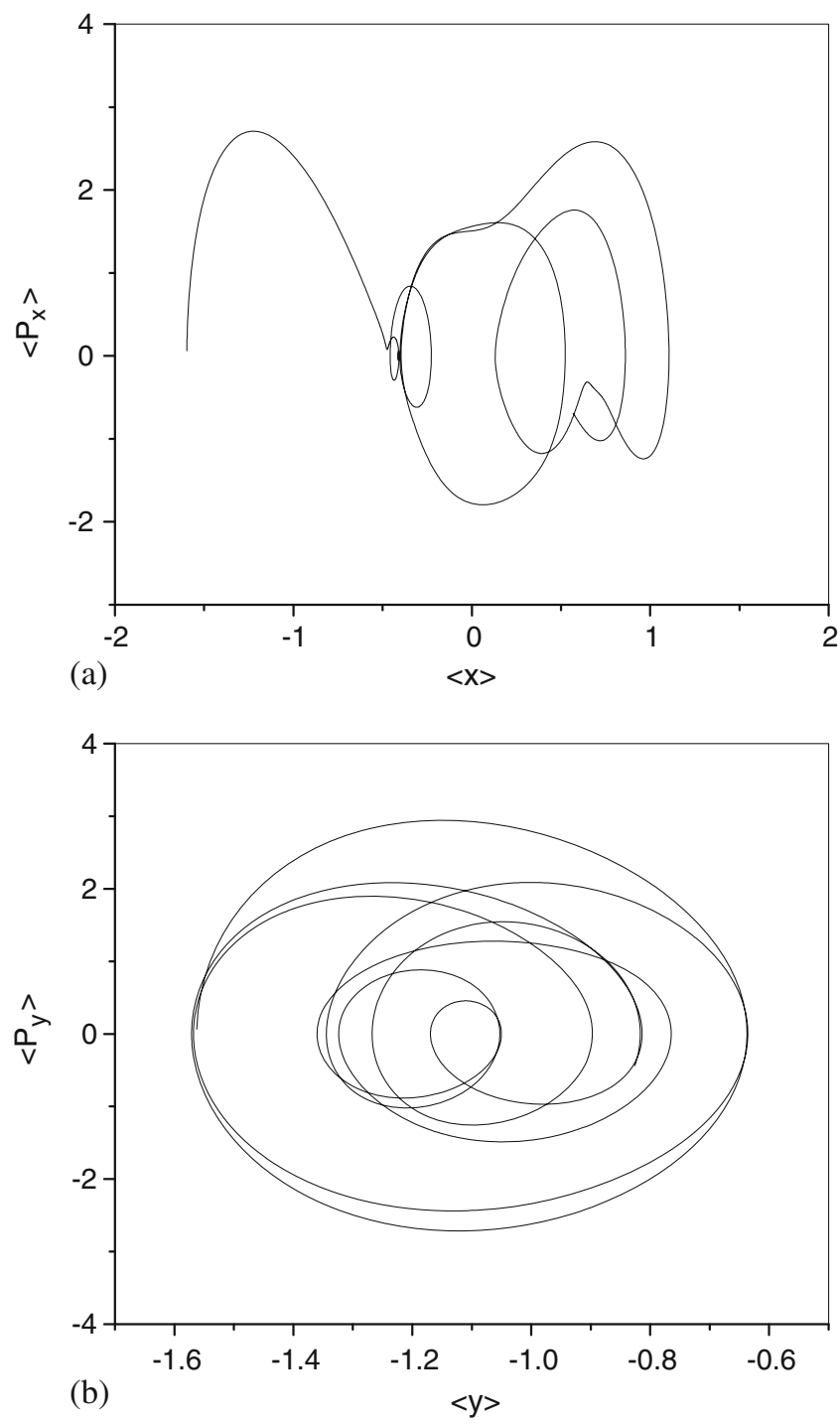

Figure 3. (a) 'Quantum phase space' diagram along the cis-cis reaction coordinate. (b) 'Quantum phase space' diagram along the cis-trans reaction coordinate. 

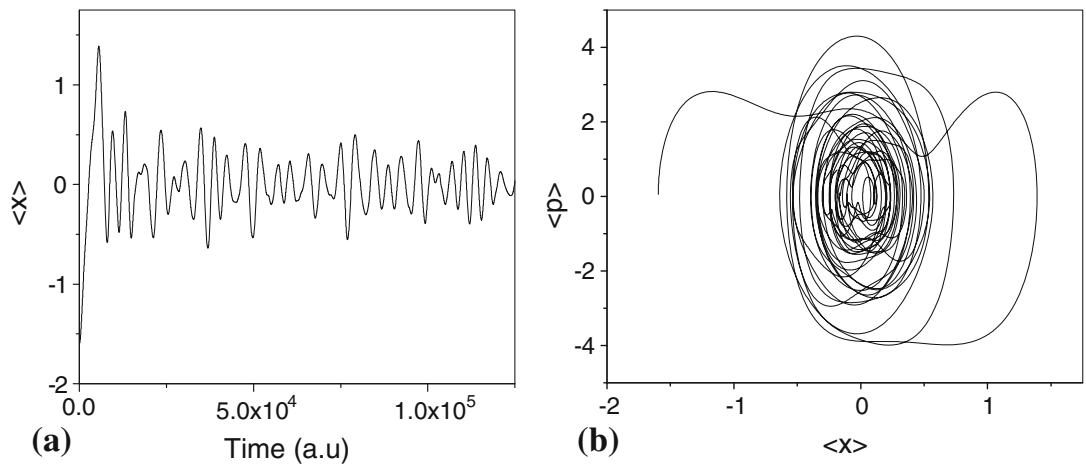

Figure 4. (a) Plot of $\langle x(t)>$ versus time showing the dynamics of the ciscis isomerization when cis-trans mode is locally excited. (b) 'Quantum phase space diagram' along cis-cis coordinate when cis-trans mode is locally excited.

takes place. The other channel, viz. cis $\leftrightarrow$ trans is virtually closed. We stress here that unless both the in-plane and out-of-plane modes are taken into account, neither the tunnelling splitting data, nor the the correct picture of the dynamics of the ground state cis-cis isomerization by tunnelling are obtained. The tunnelling transfer of $\mathrm{H}$-atom in malonaldehyde is therefore an essentially two-dimensional process.

\subsection{Controlling tunnelling $H$-atom transfer}

Control of tunnelling is an important problem and has attracted good deal of recent attention. ${ }^{19-22}$ We have investigated how the cis-cis tunnelling can be possibly quenched or attenuated by manipulating the cis-trans tunnelling mode with an external time varying field. We have locally excited the asymmetric cis-trans modes and calculated how the cis-cis tunnelling $\mathrm{H}$-atom transfer mode responds to it. Figure 4a shows how the average value of the tunnelling coordinate $(\langle x\rangle)$ evolves with time, when the asymmetric mode is coupled to a sinusoidal field of frequency $\omega=0.001$ (a.u), intensity $\epsilon_{0}=0.01$ (a.u) and phase $\delta=0$. It is clearly seen that the particle tends to get localized in the barrier region indicating that the local excitation in the asymmetric cis-trans mode coupled to the symmetric cis-cis mode tends to quench the cis-cis tunnelling isomerization. The corresponding quantum phase space diagram (figure 4b) corroborates the assertion. It would be interesting to have experimental confirmation of the prediction. When the external field is coupled to the ciscis tunnelling coordinate $(x)$ (intensity $\epsilon_{0}=0.01$ a.u., $\omega=\frac{\Delta E_{1 \rightarrow 3}}{\hbar}$, phase $\delta=0$ ), the tunnelling rate is enhanced as can be seen from the plot of $\langle x\rangle$ against time (figure 5a). The corresponding 'quantum phase space' diagram (figure 5b) also supports the enhancement of tunnelling. There is no significant change in the dynamics of cis-trans tunnelling mode whatsoever. In all the cases reported here we have verified that $E(t)$ is less than the barrier top energy so that the isomerization takes place by tunnelling only.
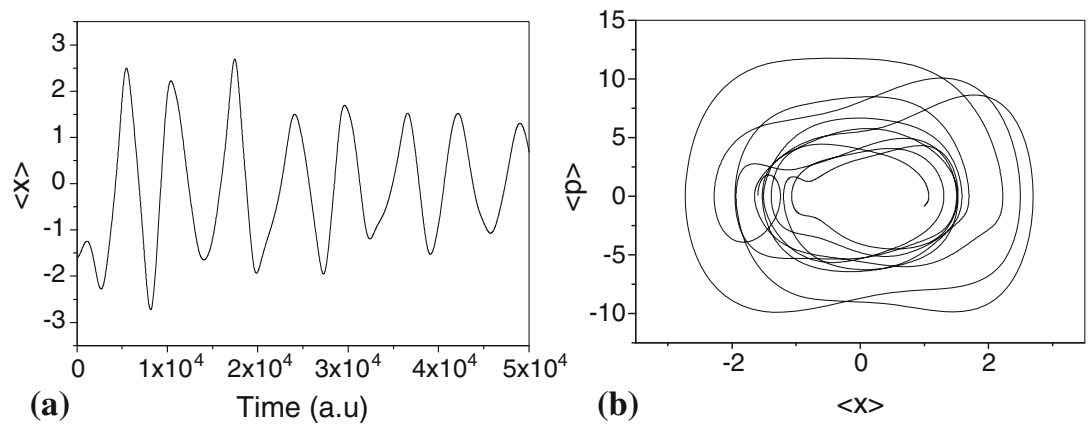

Figure 5. (a) Plot of $<x>$ versus time showing the dynamics of cis-cis isomerization when this mode is locally excited. (b) 'Quantum phase space diagram' along the cis-cis isomerization coordinate when cis-cis mode is locally excited. 


\section{Conclusion}

It can be concluded that tunnelling in a symmetrical double well may be affected by coupling with other orthogonal modes. The effect of coupling on the tunnelling rate (supression or enhancement) depends on the shape of the two-dimensional potential energy surface on which tunnelling takes place. In the case of malonaldehyde, the experimental tunnelling splitting can be reproduced only if the corresponding out-of-plane cis-trans motion of the $\mathrm{H}$-atom is taken into account along with the cis-cis motion of the transferring $\mathrm{H}$-atom and a specific form of interaction potential is assumed. The model PES proposed by us for malonaldehyde seems to describe the tunnelling dynamics quite well and could be used to describe the dynamics of $\mathrm{H}$-atom transfer in related systems.

\section{Acknowledgement}

The first author SG would like to thank the Council of Scientific and Industrial Research (CSIR), Government of India, New Delhi, for the award of a Senior Research Fellowship.

\section{References}

1. Bell R P 1980 The tunneling effect in chemistry (London: Chapman \& Hall)

2. Miyazaki T (Ed) 2003 Atom tunneling phenomena in physics, chemistry and biology (Berlin: Springer)

3. Kagan Yu and Leggett A J (eds) 1992 Quantum tunneling in condensed media (North Holland, Amsterdam)
4. Goldanskii, Trakhtenberg L I and Fleurov V N 1989 tunneling phenomena in chemical physics V. I. (New York: Gordon and Breach Science Publishers)

5. Benderskii V A, Makarov D E and Wight C A 1994 Chemical dynamics at low temperatures (New York: Wiley, John \& Sons)

6. Sekiya H, Nagashima Y and Nishimura Y 1992 J. Chem. Phys. 925761

7. Baba T, Tanaka T, Morino I, Yamada K M T and Tanaka K 1999 J. Chem. Phys. 1104131

8. Barbara P F, Walsh P K and Brus L E 1989 J. Phys. Chem. 9329

9. Douhal A, Lahmani F and Zehnacker-Rentien A 1993 Chem. Phys. 178493

10. Ensminger F A, Plassard J and Zwier T S 1993 J. Phys. Chem. 974344

11. Ghosh S and Bhattacharyya S P 2010 Tunneling dynamics and its significance in coupled systems; in Quantum frontiers of atoms and molecules (ed) Mihai V Putz (Nova Science Publishers) pp. 91-110

12. Marston C C and Baliant Kurti G B 1989 J. Chem. Phys. 913591

13. Adhikari S, Dutta P and Bhattacharyya S P 1992 Chem. Phys. Lett. 199574

14. Adhikari S, Datta P and Bhattacharyya S P 1995 Int. J. Quant. Chem. 59109

15. Hayes E F and Parr R G 1965 J. Chem. Phys. 431831

16. Epstein S T 1974 in The variational method in quantum chemistry (AP New York) p. 250

17. Maji K, Mondal C K and Bhattacharyya S P 2007 tunneling time and tunneling dynamics, Int. Rev. Phys. Chem. 26 647-670

18. Yagi K, Taketsugu T and Hirao K 2004 J. Chem. Phys. 11510647

19. Liu W A and Ballentine L E 1990 Phys. Rev. Let. 65 2927

20. Shao J S and Hangii P 1997 Phys. Rev. A 56 R4397

21. Lu W T and Wang S J 2010 Chem Phys. 36893

22. Lu G, Hai W, Zhong H and Xil Q 2010 Phys. Rev. A 81 063423 